\title{
Optimal Tuning of PID Controller for DC Motor using Bio-Inspired Algorithms
}

\author{
Nitish Katal \\ Student \\ Department of ECE, ASET \\ Amity University, Rajasthan, India.
}

\author{
Sanjay Kr. Singh \\ Associate Professor \\ Dept. of ECE, Anand International College of \\ Engineering, Jaipur, Rajasthan, India.
}

\begin{abstract}
This paper presents the performance comparison between the various soft computing techniques used for optimization of the PID controllers, implemented for speed control system for a DC motor. PID controllers are extensively used in industrial control because of their simplicity and robustness, but when industrial control is imperilled by external glitches, leads to the instability of the system. PID controller optimization using soft-computing algorithms lays emphases on obtaining the best possible PID parameters for improving the stability of the system. The PID controller has been implemented for speed control of a DC motor and the results obtained from optimization using soft-computing are compared with the ones derived from the Ziegler-Nichols method, and comparatively better results are obtained in Stimulated Annealing case.
\end{abstract}

\section{Keywords}

PID Controllers, Controller Optimization, DC Motor, Genetic Algorithms, Stimulated Annealing, Multi-objective Genetic Algorithms

\section{INTRODUCTION}

Since there is a tremendous development in the power electronic systems, yet the direct current machines are the prime source for the generation of the electric traction. Now a days, finding more useful applications in automobile industry in case of electric vehicles. Since, in DC motors, by adjusting the terminal voltage we can operate it over a wide range of speeds, thus making them compatible with most mechanical loads by virtue of their torque/speed characteristics, thus delivering high performance and easy controllability [1, 2]. But, in real time applications, there are certain factors like external noise, variable and uncertain inputs, unknown parameters, changes in the dynamics of the load, etc.; leading to the instability in their control.

PID controllers cause of their simplicity and robustness finds applications in $90 \%$ of the control systems in use today. So, the optimization of the PID controller parameters is one of the most important fields in implementation and designing of PID controllers. The classical and widely accepted method for tuning the PID parameters is computation by Ziegler-Nichols [3] method. However, computing the gains doesn't always provides the best parameters because tuning criterion presumes one-fourth reduction in the first two peaks [4]. But in real time applications, because of the noise, the tuned parameters does not always give the best results, so need is there to even fine tune them, so that they can easily adapt with these changing system dynamics. For better adaptive response of the system, in presence of external glitches, the use of various soft computing techniques like Fuzzy-Logic, Artificial Neural Networks, Genetic Algorithms, Particle Swarm
Optimization, Neuro Fuzzy, Neuro-Genetic, etc. have ceded better results.

In this paper, the optimization of the PID controller gains has been carried out using Genetic Algorithms (GA), MultiObjective Genetic Algorithms (Mobj-GA) and Stimulated Annealing, while using the Ziegler-Nichols parameters for the determination of the lower and upper bound limits for the initialization of PID parameter populations.

Then, the optimization of the PID controllers for the estimation of the best PID parameters has been done with respect to the objective function, stated as, "Sum of the integral of the squared error and the squared controller output deviated from its steady-state"

According to the results obtained in this paper, considerably better results have been obtained in the case of the Stimulated Annealing, when compared to the other techniques in respect of the step response of the system.

\section{PID Controllers}

Proportional Integral and Derivative -PID controllers because of their simplicity and wide acceptability, are playing an imperative role in control systems, and for regulating the closed loop response in industrial controls, PID controllers alone contribute $90 \%$ of all the PID's used today. A PID controller based system is represented in simple block level diagram as in Figure 1.

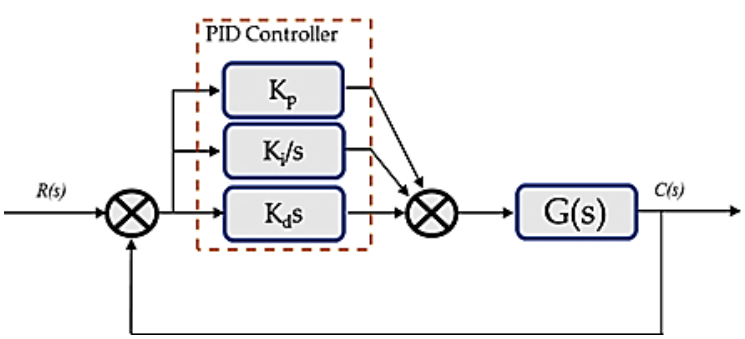

Figure 1. Schematic representation of unity feedback PID controller system architecture.

The general equation for a PID controller for the above figure can be given as [5]:

$$
C(s)=K_{p} \cdot R(s)+K_{i} \int R(s) d t+K_{d} \frac{d R(s)}{d t}
$$

Where $K_{p}, K_{i}$ and $K_{d}$ are the controller gains, $C(s)$ is output signal, $R(s)$ is the difference between the desired output and output obtained.

Some of the prime methods for tuning are: Mathematical criteria, Cohen-Coon Method, Trail and Error Method, Ziegler-Nichols Method and now a days the Soft-Computing 
techniques, being lesser prone to error when compared to conventional methods; like Fuzzy Logic, Genetic Algorithms, Particle Swarm Optimization, Neuro-Fuzzy, Simulated Annealing and Artificial Neural Networks, are also becoming dominant in research methodologies.

\section{Mathematical Modeling of DC Motor}

The simple mathematical model of a DC Motor is shown in Figure 2. The calculation of the transfer function of the motor has been done using the various parameters listed below as:

- Moment of Inertia $(\mathrm{J})=0.02 \mathrm{~kg} \cdot \mathrm{m}^{2} / \mathrm{s}^{2}$

- Damping Ratio of Mechanical System (b) $=0.3 \mathrm{Nms}$

- Electromotive Force Constant $(\mathrm{K}=\mathrm{Ke}=\mathrm{Kt}) \quad=0.04$ $\mathrm{Nm} /$ Amps

- Electric Resistance $(\mathrm{R})=4 \mathrm{ohm}$

- Electric Inductance $(\mathrm{L})=0.5 \mathrm{H}$

- Input Voltage $(\mathrm{V})=$ Source Voltage

- Output $=$ Position of Shaft .

In the Figure 2, the resistance of the circuit is denoted by $R$ and the inductance by $L, V_{a p p}$ is the voltage applied and the $\mathrm{i}(t)$ is the current; $\theta$ is angular position of the shaft.

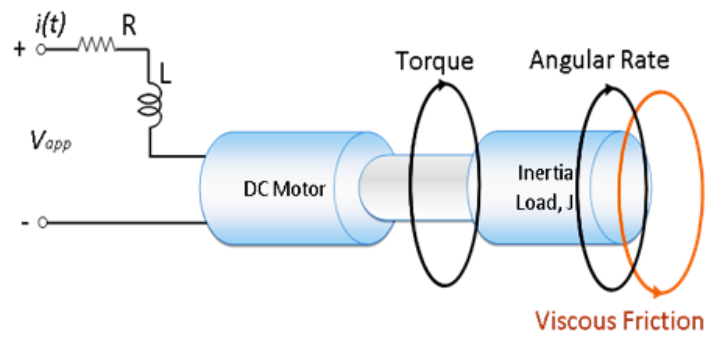

Figure 2: Schematic Representation of a DC Motor

The transfer function of the motor is calculated as per the differential equations of the motor, and the transfer function can be represented as:

$$
\begin{aligned}
& G(s)= \\
& \frac{K}{(J * L) \cdot s^{2}+((J * R)+(L * b)) \cdot s+\left(\left(b^{*} R\right)+K^{2}\right)}
\end{aligned}
$$

Using the values of the parameters of DC motor in above transfer function, the value of $G(s)$ is obtained as:

$$
G(s)=\frac{0.04}{0.01 \cdot s^{2}+0.23 \cdot s+1.202}
$$

\section{Designing \& Tuning of PID Controllers}

\subsection{PID Tuning using Ziegler-Nichols}

One of the most operative methods of tuning of the PID controllers is to use the open loop response as implied by Ziegler-Nichols, yet this method is limited for application till the ratio of 4:1 for the first two peaks in the closed loop response [5], which leads to the oscillatory response of the system.

Initially the unit step function (Figure 3) is derived, and hence as suggested by Ziegler-Nichols, the parameters required for the implementation of the PID controllers can be easily estimated as shown in Table 1.

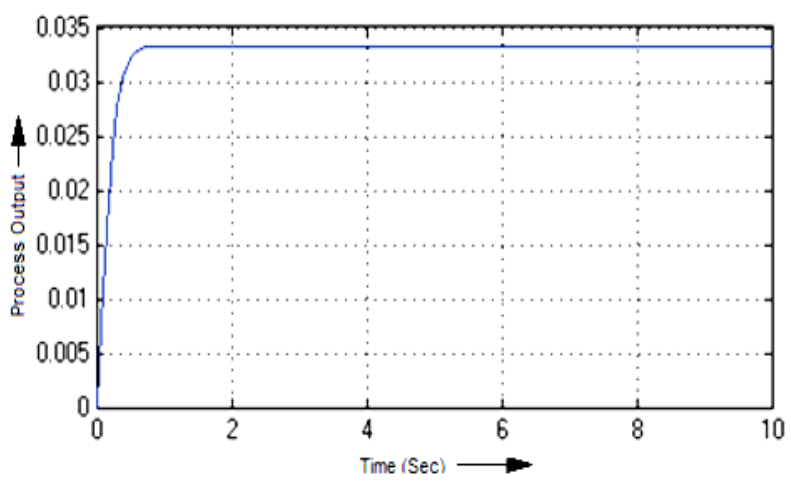

Figure 3: Open Loop Response of the system

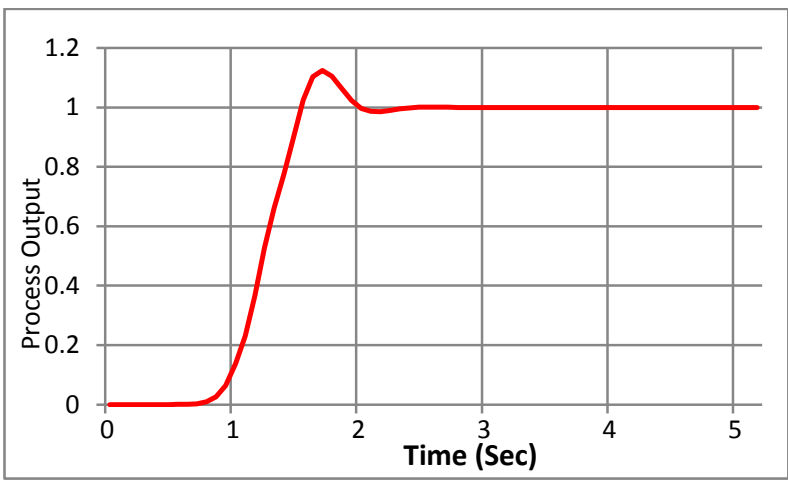

Figure 4: Closed loop ZN-PID response of the system

Table 1. Parameters of the PID Controller Calculated by Ziegler Nichols.

\begin{tabular}{|l|l|}
\hline Ziegler-Nichols PID Parameters & Value \\
\hline$K p$ & 12.63 \\
\hline$K i$ & 191.361 \\
\hline$K d$ & 0.2083 \\
\hline
\end{tabular}

\subsection{PID Optimization using Genetic}

\section{Algorithm}

Since the Ziegler-Nichols based PID controllers gives an oscillatory response, so the PID parameters are not optimum for direct implementation for the plant. So their organized optimization is must, so that better parameters can be estimated and when applied to the system, delivers best performance and robustness.

Genetic Algorithm provides a solution for the optimization of the PID controllers, by minimizing the objective function. The parameters obtained by Ziegler-Nichols method are used for the initial declaration for the parameter population, in order to achieve faster convergence. The various steps involved in optimization using Genetic Algorithms are as:

1. Generation of initial random population of fixed number of individuals for the initial ranges if $K p, K i$ and $K d$.

2. Evaluation of fitness integral, it minimizes the integral square error, followed by the selection of the fittest individuals.

3. Reproduction among the members of the population. 
4. Crossover of the reproduced chromosome, followed by mutation operation, and the selection of the best individuals i.e. Survival of the Fittest.

5. Looping the step 2 till the pre-defined convergence is obtained.

The optimization of the system has been designed and simulated in Matlab and Simulink environment and the optimization has been carried out using Global Optimization Toolbox. The various, parameters, used in Genetic Algorithm are, 100 population sizes, scattered crossover, migration in both directions, and roulette wheel based selection function.

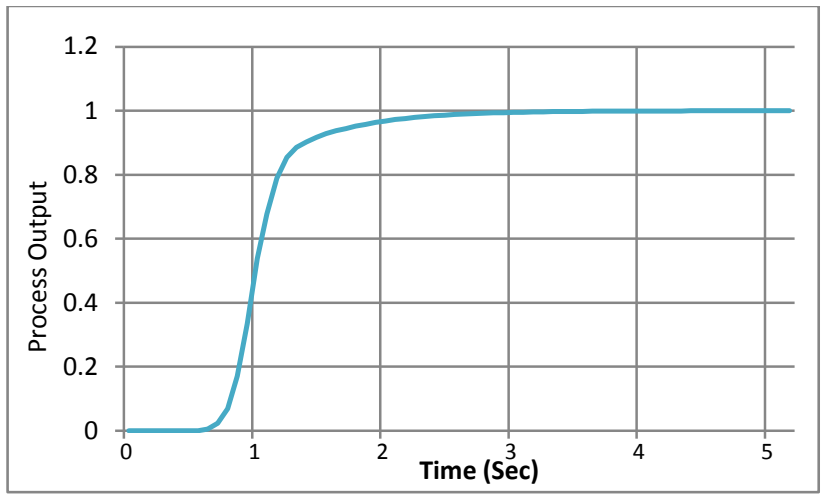

Figure 5: Closed loop response of the system using GA optimized PID controllers

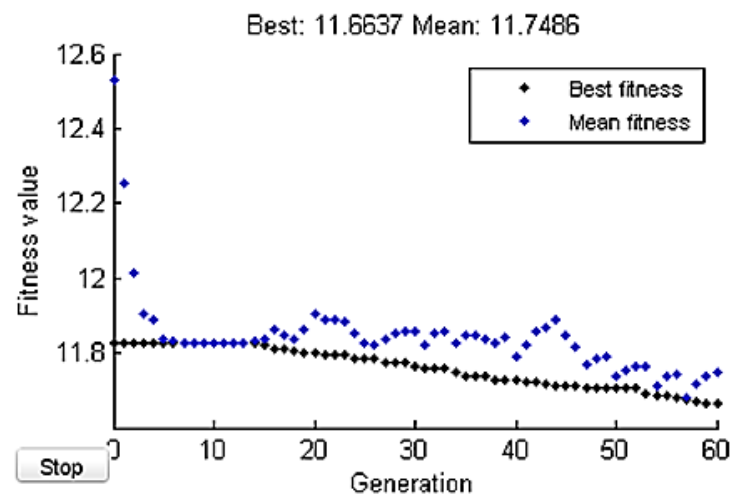

Figure 6: Plot for the best and average fitness value of the genetic algorithm optimization.

Table 2. Parameters of the PID Controller optimized by Genetic Algorithm.

\begin{tabular}{|l|l|}
\hline Genetic Algorithm PID Parameters & Value \\
\hline $\mathrm{Kp}$ & 119.5071 \\
\hline $\mathrm{Ki}$ & 187.0221 \\
\hline $\mathrm{Kd}$ & 5.6306 \\
\hline
\end{tabular}

\subsection{PID Optimization using Multi- Objective Genetic Algorithm}

Optimization of the PID's using multi-objective genetic algorithm aims at using the controlled elitist genetic algorithm which boosts obtaining the better fitness value of the individuals and if the fitness value is less, it still favours increasing the diversity of the population [6]. Diversity is controlled by the elite members of the population; elitism is controlled by Pareto fraction and at Pareto Front also bound the number of individuals. The parameter determined by $\mathrm{ZN}$ helps in the determination of the initial lower and upper bound limits to be used for the optimization, and focuses on minimizing the integral square error.

The system implementation and optimization has been carried out in Matlab and Simulink environment using Global Optimization Toolbox. The population size of 45 has been considered, with adaptive feasible mutation function, heuristic crossover and the selection of individuals on the basis of tournament with a tournament size of 2 . A hybrid function of Fitness Goal Attain (fgoalattain) is used which further minimizes the function after GA terminates. After the optimization the PID parameters are shown in Table 3 along with the controller response in figure 7.

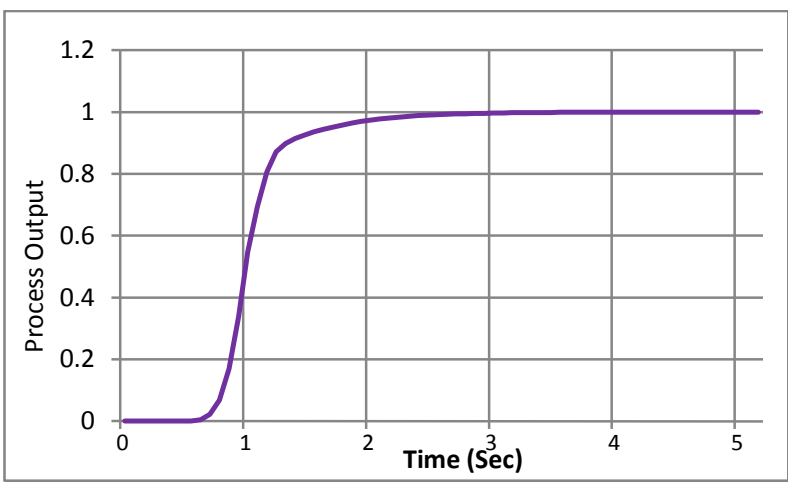

Figure 7: Closed loop response of the system using MobjGA optimized PID controllers
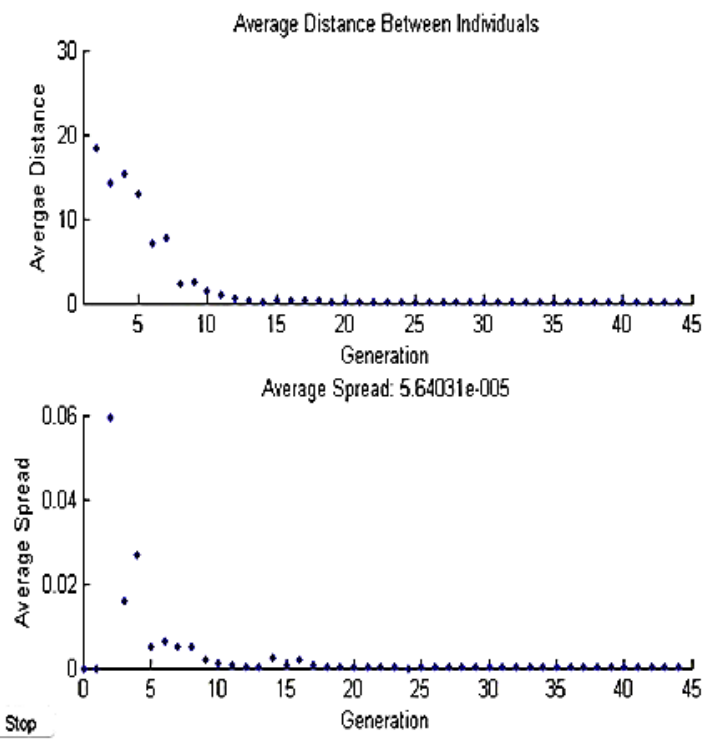

Fig 8:(1) Plot for average distance between individuals.

(2) Plot for the average spread between individuals

Table 3. Parameters of the PID Controller optimized by Multi-Objective Genetic Algorithm.

\begin{tabular}{|l|l|}
\hline Mobj-GA PID Parameters & Value \\
\hline $\mathrm{Kp}$ & 116.3364 \\
\hline $\mathrm{Ki}$ & 204.7561 \\
\hline $\mathrm{Kd}$ & 5 \\
\hline
\end{tabular}




\subsection{PID Controller Optimization using Simulated Annealing}

Stimulated annealing is a global optimization algorithm, as the name suggests, the muse comes from metallurgic annealing, which involves relation between the relation between the statical mechanics and multivariate optimization [7]. It follows the technique involving heating the material followed by controlled cooling, fetching increased crystal size and reduced deformities.

In stimulated annealing (SA), at each iteration, a new point is randomly generated and its distance from the current point is the function of probability distribution with a scale proportional to temperature. The randomly generated points are accepted if they lower the objective but in order to help the algorithm to search for global solution and to omit the trapping of the algorithm in local minima, some points are so chosen that they raise the objective. With the advent of algorithm, the temperature is decreased leading in reduction of the extent of search to converge towards the minima.

The optimization has been carried out in MATLAB and Simulink environment with the help of Global Optimization Toolbox using Stimulated Annealing function. For stimulated annealing, the Boltzmann Annealing function has been used with an exponentially updating temperature. The parameters obtained after optimization are in table 4 and the response of the PID controller with SA optimised parameters is shown in figure 9. Figure 10 shows the Plot for the Best function value of the Stimulated Annealing optimization.

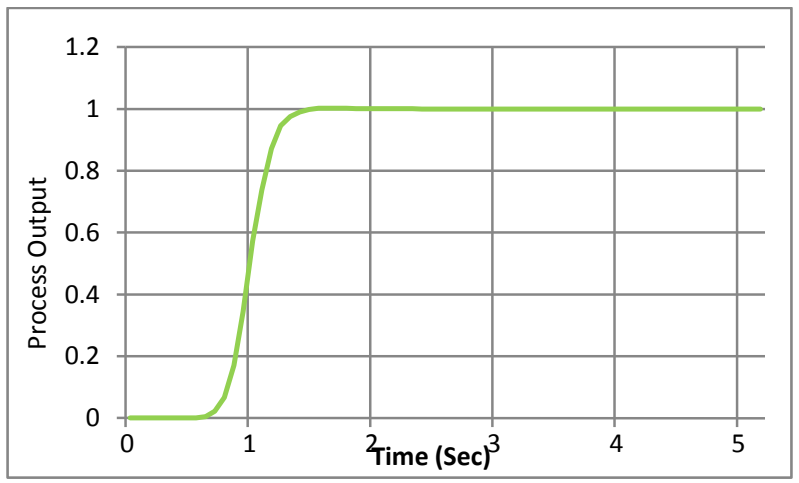

Figure 9: Closed loop response of the system using Stimulated Annealing optimized PID controllers

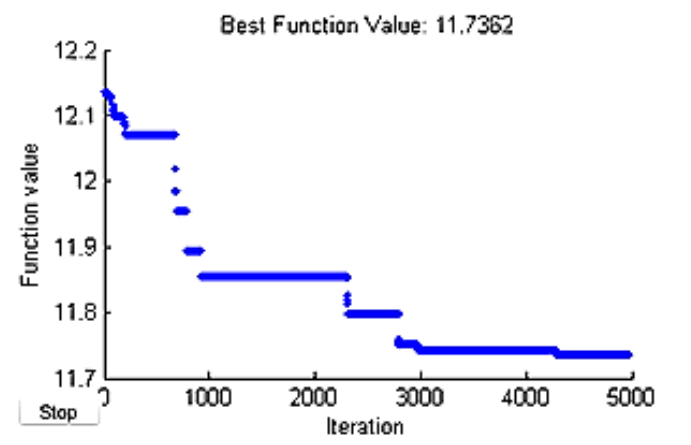

Figure 10: Plot for the Best function value of the Stimulated Annealing optimization.
Table 4: Parameters of the PID Controller optimized by Multi-Objective Stimulated Annealing.

\begin{tabular}{|l|l|}
\hline $\begin{array}{l}\text { Stimulated Annealing PID } \\
\text { Parameters }\end{array}$ & Value \\
\hline$K p$ & 112.5936 \\
\hline$K i$ & 353.4372 \\
\hline$K d$ & 4.5 \\
\hline
\end{tabular}

\section{Results \& Discussion}

In this paper, a Simulink model of a DC motor has been designed and for controlling the speed, a PID controller based system has been designed; followed by the estimation of PID parameters of $K p K i$ and $K d$ using Ziegler-Nichols method. Since the ZN-PID controllers when implemented for the systems, presents an oscillatory response, so the optimization of the PID gains is carried out by various softcomputing techniques like Genetic Algorithms, MultiObjective Genetic Algorithm and Stimulated Annealing. The parameters obtained by Ziegler-Nichols have been used as boundary limits for the PID controller optimization populations to provide, faster convergence.

The computed parameters are implemented for the closedloop response of the system. It is clearly evident from the Figure 10 and Figure 11 that the stimulated annealing solutions present best values rise and settling times but slight overshoot is obtained in the response. The results are presented in tabular form (Table 5).

Concluding, Stimulated Annealing offers superior results in terms of controller output and system performance for the tuning of PID controllers when the values are compared in Figure 10 and 11 and Table 5.

Table 5. Comparison of the Results.

\begin{tabular}{|l|c|c|c|}
\hline \multicolumn{1}{|c|}{ Method of Design } & $\begin{array}{c}\text { Overshoot } \\
\text { Percentage }\end{array}$ & $\begin{array}{c}\text { Rise } \\
\text { Time } \\
\text { (Sec) }\end{array}$ & $\begin{array}{c}\text { Settling } \\
\text { Time } \\
\text { (Sec) }\end{array}$ \\
\hline Ziegler-Nichols & $12.37 \%$ & 0.253 & 0.849 \\
\hline Genetic Algorithms & $0 \%$ & 0.243 & 1.274 \\
\hline $\begin{array}{l}\text { Multi-Objective } \\
\text { Genetic Algorithms }\end{array}$ & $0 \%$ & 0.214 & 1.112 \\
\hline Stimulated Annealing & $0.27 \%$ & 0.145 & 0.248 \\
\hline
\end{tabular}

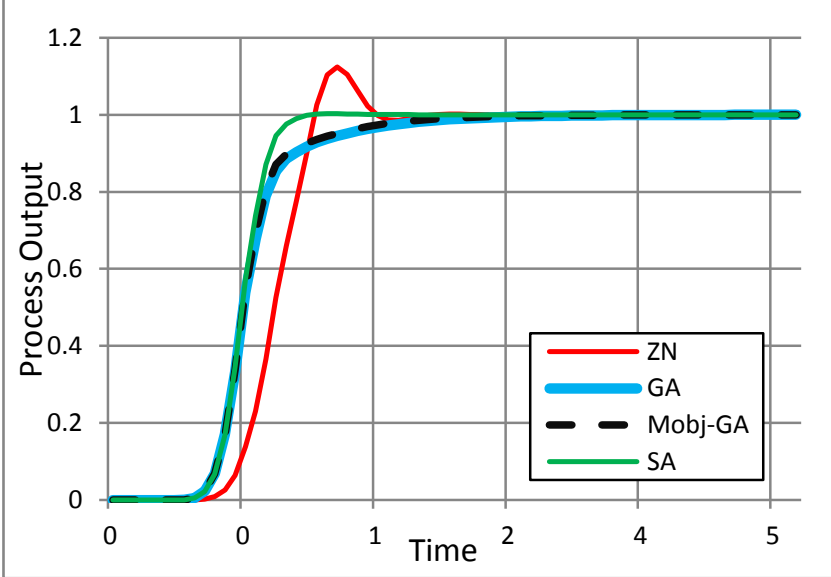

Figure 11: Compared closed loop step response of the ZN, SISO \& GA-PID Controllers. 


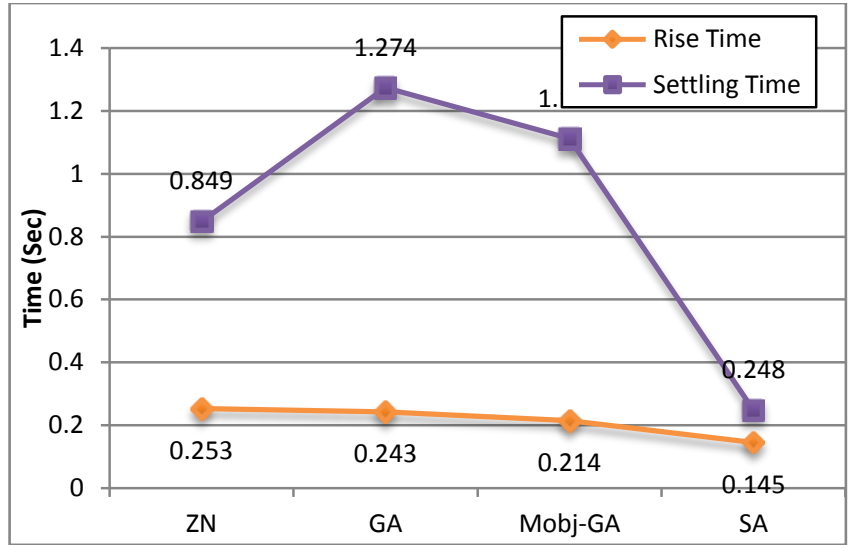

Figure 12: Compared variation in rise and settling times of the ZN, SISO \& GA-PID Controllers.

\section{Conclusion}

The use of Stimulated Annealing for optimizing the PID controller parameters as presented in this paper offers advantages of tremendously decreased overshoot percentage, rise and settling times for the designed DC motor. Simulation results when compared with the other tuning methodologies as presented in this paper, Stimulated Annealing has proved effective in achieving the steady-state response and performance indices.

\section{References}

[1] Wang, J. B., Control of Electric Machinery. Gau Lih Book co., Ltd, Taipei Taiwan, 2001.
[2] G. Haung and S. Lee, "PC based PID speed control in DC motor," IEEE Conf. SALIP-2008, pp. 400-408, 2008.

[3] Ziegler, J.G and Nichols, N. B. (1942). "Optimum settings for automatic controllers." Transactions of the ASME. 64. pp. 759-768.

[4] Goodwin, G. C., Graebe, S. F. and Salgado, M.E. 2001 Control System Design, Prentice Hall Inc., New Jersey.

[5] Norman S. Nise, 2003 , Control System Engineering, 4th Edition,

[6] Deb, Kalyanmoy. "Multi-Objective Optimization Using Evolutionary Algorithms.” John Wiley \& Sons, 2001.

[7] Kickpatrik S., Gelatt C.D., Vecchi M.P. (1983), "Optimization by Stimulated Annealing”, Science (220) 4508, p-671-680,

[8] Granville, V.; Krivanek, M.; Rasson, J.-P. (1994). "Simulated annealing: A proof of convergence". IEEE Transactions on Pattern Analysis and Machine Intelligence 16

[9] Abdullah Konak, David W. Coit, Alice E. Smith , "Multiobjective optimization using genetic algorithm", Reliability Engineering and Safety System, 91 (2006) 992-1007, Elsevier Ltd.

[10] Berrsimas D., Tsitsiklis, “ Stimulated Annealing” (1993), Statistical Science, Vol. 8, No. 1, 10-15

[11] MATLAB and SIMULINK Documentation 